\title{
A note on the existence of $H$-bubbles via perturbation methods
}

\section{Veronica Felli}

\begin{abstract}
We study the problem of existence of surfaces in $\mathbb{R}^{3}$ parametrized on the sphere $\mathbb{S}^{2}$ with prescribed mean curvature $H$ in the perturbative case, i.e. for $H=H_{0}+\varepsilon H_{1}$, where $H_{0}$ is a nonzero constant, $H_{1}$ is a $C^{2}$ function and $\varepsilon$ is a small perturbation parameter.
\end{abstract}

\section{Introduction}

In this paper we are interested in the existence of $H$-bubbles, namely of $\mathbb{S}^{2}$-type parametric surfaces in $\mathbb{R}^{3}$ with prescribed mean curvature $H$. This geometrical problem is motivated by some models describing capillarity phenomena and has the following analytical formulation: given a function $H \in C^{1}\left(\mathbb{R}^{3}\right)$, find a smooth nonconstant function $\omega: \mathbb{R}^{2} \rightarrow \mathbb{R}^{3}$ which is conformal as a map on $\mathbb{S}^{2}$ and solves the problem

$\left(P_{H}\right)$

$$
\left\{\begin{array}{l}
\Delta \omega=2 H(\omega) \omega_{x} \wedge \omega_{y}, \quad \text { in } \mathbb{R}^{2}, \\
\int_{\mathbb{R}^{2}}|\nabla \omega|^{2}<+\infty,
\end{array}\right.
$$

where

$$
\begin{aligned}
\omega_{x} & =\left(\frac{\partial \omega_{1}}{\partial x}, \frac{\partial \omega_{2}}{\partial x}, \frac{\partial \omega_{3}}{\partial x}\right), & \omega_{y} & =\left(\frac{\partial \omega_{1}}{\partial y}, \frac{\partial \omega_{2}}{\partial y}, \frac{\partial \omega_{3}}{\partial y}\right), \\
\Delta \omega & =\omega_{x x}+\omega_{y y}, & \nabla \omega & =\left(\omega_{x}, \omega_{y}\right),
\end{aligned}
$$

and $\wedge$ denotes the exterior product in $\mathbb{R}^{3}$.

Brezis and Coron [4] proved that for constant nonzero mean curvature $H(u) \equiv H_{0}$ the only nonconstant solutions are spheres of radius $\left|H_{0}\right|^{-1}$.

2000 Mathematics Subject Classification: 53A10, 35J50, 35B20.

Keywords: H-surfaces, nonlinear elliptic systems, perturbative methods. 
While the Plateau and the Dirichlet problems have been largely studied both for $H$ constant and for $H$ nonconstant (see $[3,4,10,12,13,14$, $15,16])$, problem $\left(P_{H}\right)$ in the case of nonconstant $H$ has been investigated only recently, see $[5,6,7]$. In [5] Caldiroli and Musina proved the existence of $H$-bubbles with minimal energy under the assumptions that $H \in C^{1}\left(\mathbb{R}^{3}\right)$ satisfies

$$
\begin{aligned}
& \text { (i) } \sup _{u \in \mathbb{R}^{3}}|\nabla H(u+\xi) \cdot u u|<1 \quad \text { for some } \xi \in \mathbb{R}^{3} \text {, } \\
& \text { (ii) } H(u) \rightarrow H_{\infty} \quad \text { as }|u| \rightarrow \infty \quad \text { for some } H_{\infty} \in \mathbb{R} \\
& \text { (iii) } \quad c_{H}=\inf _{\substack{u \in C_{c}^{1}\left(\mathbb{R}^{2}, \mathbb{R}^{3}\right) \\
u \neq 0}} \sup _{s>0} \mathcal{E}_{H}(s u)<\frac{4 \pi}{3 H_{\infty}^{2}}
\end{aligned}
$$

where

$$
\mathcal{E}_{H}(u)=\frac{1}{2} \int_{\mathbb{R}^{2}}|\nabla u|^{2}+2 \int_{\mathbb{R}^{2}} Q(u) \cdot u_{x} \wedge u_{y}
$$

and $Q: \mathbb{R}^{3} \rightarrow \mathbb{R}^{3}$ is any vector field such that $\operatorname{div} Q=H$.

The perturbative method introduced by Ambrosetti and Badiale [1,2] was used in [7] to treat the case in which $H$ is a small perturbation of a constant, namely

$$
H(u)=H_{\varepsilon}(u)=H_{0}+\varepsilon H_{1}(u),
$$

where $H_{0} \in \mathbb{R} \backslash\{0\}, H_{1} \in C^{2}\left(\mathbb{R}^{3}\right)$, and $\varepsilon$ is a small real parameter. This method allows to find critical points of a functional $f_{\varepsilon}$ of the type $f_{\varepsilon}(u)=$ $f_{0}(u)-\varepsilon G(u)$ in a Banach space by studying a finite dimensional problem. More precisely, if the unperturbed functional $f_{0}$ has a finite dimensional manifold of critical points $Z$ which satisfies a nondegeneracy condition, it is possible to prove, for $|\varepsilon|$ sufficiently small, the existence of a smooth function $\eta_{\varepsilon}(z): Z \rightarrow\left(T_{z} Z\right)^{\perp}$ such that any critical point $\bar{z} \in Z$ of the function

$$
\Phi_{\varepsilon}: Z \rightarrow \mathbb{R}, \quad \Phi_{\varepsilon}(z)=f_{\varepsilon}\left(z+\eta_{\varepsilon}(z)\right)
$$

gives rise to a critical point $u_{\varepsilon}=\bar{z}+\eta_{\varepsilon}(\bar{z})$ of $f_{\varepsilon}$, i.e. the perturbed manifold $Z_{\varepsilon}:=\left\{z+\eta_{\varepsilon}(z): z \in Z\right\}$ is a natural constraint for $f_{\varepsilon}$. Furthermore $\Phi_{\varepsilon}$ can be expanded as

$$
\Phi_{\varepsilon}(z)=b-\varepsilon \Gamma(z)+o(\varepsilon) \quad \text { as } \varepsilon \rightarrow 0
$$

where $b=f_{0}(z)$ and $\Gamma$ is the Melnikov function defined as the restriction of the perturbation $G$ on $Z$, namely $\Gamma=\left.G\right|_{Z}$. For problem $\left(P_{H_{\varepsilon}}\right), \Gamma$ is given by

$$
\Gamma: \mathbb{R}^{3} \rightarrow \mathbb{R}, \quad \Gamma(p)=\int_{|p-q|<\frac{1}{\left|H_{0}\right|}} H_{1}(q) d q
$$


In [7] Caldiroli and Musina studied the functional $\Gamma$ giving some existence results in the perturbative setting for problem $\left(P_{H_{\varepsilon}}\right)$. They prove that for $|\varepsilon|$ small there exists a smooth $H_{\varepsilon}$-bubble if one of the following conditions holds

1) $H_{1}$ has a nondegenerate stationary point and $\left|H_{0}\right|$ is large;

2) $\max _{p \in \partial K} H_{1}(p)<\max _{p \in K} H_{1}(p)$ or $\min _{p \in \partial K} H_{1}(p)>\min _{p \in K} H_{1}(p)$ for some nonempty compact set $K \subset \mathbb{R}^{3}$ and $\left|H_{0}\right|$ is large;

3) $H_{1} \in L^{r}\left(\mathbb{R}^{3}\right)$ for some $r \in[1,2]$.

They prove that critical points of $\Gamma$ give rise to solutions to $\left(P_{H_{\varepsilon}}\right)$ for $\varepsilon$ sufficiently small. Precisely the assumption that $H_{0}$ is large required in cases 1) and 2) ensures that if $H_{1}$ is not constant then $\Gamma$ is not identically constant. If we let this assumption drop, it may happen that $\Gamma$ is constant even if $H_{1}$ is not. This fact produces some loss of information because the first order expansion (1.1) is not sufficient to deduce the existence of critical points of $\Phi_{\varepsilon}$ from the existence of critical points of $\Gamma$. Instead of studying $\Gamma$ we perform a direct study of $\Phi_{\varepsilon}$ which allows us to prove some new results. In the first one, we assume that $H_{1}$ vanishes at $\infty$ and has bounded gradient, and prove the existence of a solution without branch points. Let us recall that a branch point for a solution $\omega$ to $\left(P_{H}\right)$ is a point where $\nabla \omega=0$, i.e. a point where the surface parametrized by $\omega$ fails to be immersed.

Theorem 1.1 Let $H_{0} \in \mathbb{R} \backslash\{0\}, H_{1} \in C^{2}\left(\mathbb{R}^{3}\right)$ such that

$$
\begin{array}{ll}
(H 1) & \lim _{|p| \rightarrow \infty} H_{1}(p)=0 ; \\
(H 2) & \nabla H_{1} \in L^{\infty}\left(\mathbb{R}^{3}, \mathbb{R}^{3}\right) .
\end{array}
$$

Let $H_{\varepsilon}=H_{0}+\varepsilon H_{1}$. Then for $|\varepsilon|$ sufficiently small there exists a smooth $H_{\varepsilon}$-bubble without branch points.

With respect to case 1) of [7] we require neither nondegeneracy of critical points of $H_{1}$ nor largeness of $H_{0}$. With respect to case 2) we do not assume that $H_{0}$ is large; on the other hand our assumption (H1) implies 2). Moreover we do not assume any integrability condition of type 3 ). With respect to the result proved in [5], we have the same kind of behavior of $H_{1}$ at $\infty$ (see $(i i)$ and assumption $(H 1)$ ) but we do not need any assumption of type (iii); on the other hand in [5] it is not required that the prescribed curvature is a small perturbation of a constant.

The following results give some conditions on $H_{1}$ in order to have two or three solutions. 
Theorem 1.2 Let $H_{0} \in \mathbb{R} \backslash\{0\}, H_{1} \in C^{2}\left(\mathbb{R}^{3}\right)$ such that (H1), (H2),

(H3) Hess $H_{1}(p)$ is positive definite for any $p \in B_{1 /\left|H_{0}\right|}(0)$,

(H4) $H_{1}(p)>0$ in $B_{1 /\left|H_{0}\right|}(0)$,

hold. Then for $|\varepsilon|$ sufficiently small there exist at least three smooth $H_{\varepsilon}$-bubbles without branch points.

Remark 1.3 If we assume $(H 1)$, (H2), and, instead of $(H 3)-(H 4)$, that $H_{1}(0)>0$ and Hess $H_{1}(0)$ is positive definite, then we can prove that for $\left|H_{0}\right|$ sufficiently large and $|\varepsilon|$ sufficiently small there exist at least three smooth $H_{\varepsilon}$-bubbles without branch points.

Theorem 1.4 Let $H_{0} \in \mathbb{R} \backslash\{0\}, H_{1} \in C^{2}\left(\mathbb{R}^{3}\right)$ such that $(H 1)$ and $(H 2)$ hold. Assume that there exist $p_{1}, p_{2} \in \mathbb{R}^{3}$ such that

$$
\int_{B\left(p_{1}, 1 /\left|H_{0}\right|\right)} H_{1}(\xi) d \xi>0 \quad \text { and } \quad \int_{B\left(p_{2}, 1 /\left|H_{0}\right|\right)} H_{1}(\xi) d \xi<0 .
$$

Then for $|\varepsilon|$ sufficiently small there exist at least two smooth $H_{\varepsilon}$-bubbles without branch points.

Remark 1.5 If we assume (H1), (H2), and, instead of (H5), that there exist $p_{1}, p_{2} \in \mathbb{R}^{3}$ such that $H_{1}\left(p_{1}\right)>0$ and $H_{1}\left(p_{2}\right)<0$, then we can prove that for $\left|H_{0}\right|$ sufficiently large and $|\varepsilon|$ sufficiently small there exist at least two smooth $H_{\varepsilon}$-bubbles without branch points.

The present paper is organized as follows. In Section 2 we introduce some notation and recall some known facts whereas Section 3 is devoted to the proof of Theorems 1.1, 1.2, and 1.4.

\section{Notation and known facts}

In the sequel we will take $H_{0}=1$; this is not restrictive since we can do the change $H_{1}(u)=H_{0} \widetilde{H}_{1}\left(H_{0} u\right)$. Hence we will always write

$$
H_{\varepsilon}(u)=1+\varepsilon H(u),
$$

where $H \in C^{2}\left(\mathbb{R}^{3}\right)$. Let us denote by $\omega$ the function $\omega: \mathbb{R}^{2} \rightarrow \mathbb{S}^{2}$ defined as

$$
\omega(x, y)=(\mu(x, y) x, \mu(x, y) y, 1-\mu(x, y)), \text { where } \mu(x, y)=\frac{2}{1+x^{2}+y^{2}} .
$$

Note that $\omega$ is a conformal parametrization of the unit sphere and solves

$$
\left\{\begin{array}{l}
\Delta \omega=2 \omega_{x} \wedge \omega_{y} \quad \text { on } \mathbb{R}^{2} \\
\int_{\mathbb{R}^{2}}|\nabla \omega|^{2}<+\infty .
\end{array}\right.
$$


Problem (2.1) has in fact a family of solutions of the form $\omega \circ \phi+p$ where $p \in \mathbb{R}^{3}$ and $\phi$ is any conformal diffeomorphism of $\mathbb{R}^{2} \cup\{\infty\}$. For $s \in(1,+\infty)$, we will set $L^{s}:=L^{s}\left(\mathbb{S}^{2}, \mathbb{R}^{3}\right)$, where any map $v \in L^{s}$ is identified with the map $v \circ \omega: \mathbb{R}^{2} \rightarrow \mathbb{R}^{3}$ which satisfies

$$
\int_{\mathbb{R}^{2}}|v \circ \omega|^{s} \mu^{2}=\int_{\mathbb{S}^{2}}|v|^{s}
$$

We will use the same notation for $v$ and $v \circ \omega$. By $W^{1, s}$ we denote the Sobolev space $W^{1, s}\left(\mathbb{S}^{2}, \mathbb{R}^{3}\right)$ endowed (according to the above identification) with the norm

$$
\|v\|_{W^{1, s}}=\left[\int_{\mathbb{R}^{2}}|\nabla v|^{s} \mu^{2-s}\right]^{1 / s}+\left[\int_{\mathbb{R}^{2}}|v|^{s} \mu^{2}\right]^{1 / s} .
$$

If $s^{\prime}$ is the conjugate exponent of $s$, i.e. $1 / s+1 / s^{\prime}=1$, the duality product between $W^{1, s}$ and $W^{1, s^{\prime}}$ is given by

$$
\langle v, \varphi\rangle=\int_{\mathbb{R}^{2}} \nabla v \cdot \nabla \varphi+\int_{\mathbb{R}^{2}} v \cdot \varphi \mu^{2} \quad \text { for any } v \in W^{1, s} \text { and } \varphi \in W^{1, s^{\prime}} .
$$

Let $Q$ be any smooth vector field on $\mathbb{R}^{3}$ such that $\operatorname{div} Q=H$. The energy functional associated to problem

$$
\left\{\begin{array}{l}
\Delta u=2(1+\varepsilon H(u)) u_{x} \wedge u_{y}, \quad \text { in } \mathbb{R}^{2}, \\
\int_{\mathbb{R}^{2}}|\nabla u|^{2}<+\infty
\end{array}\right.
$$

is given by

$$
\mathcal{E}_{\varepsilon}(u)=\frac{1}{2} \int_{\mathbb{R}^{2}}|\nabla u|^{2}+2 \mathcal{V}_{1}(u)+2 \varepsilon \mathcal{V}_{H}(u), \quad u \in W^{1,3}
$$

where

$$
\mathcal{V}_{H}(u)=\int_{\mathbb{R}^{2}} Q(u) \cdot u_{x} \wedge u_{y}
$$

has the meaning of an algebraic volume enclosed by the surface parametrized by $u$ with weight $H$ (it is independent of the choice of $Q$ ); in particular

$$
\mathcal{V}_{1}(u)=\frac{1}{3} \int_{\mathbb{R}^{2}} u \cdot u_{x} \wedge u_{y}
$$

In [7], Caldiroli and Musina studied some regularity properties of $\mathcal{V}_{H}$ on the space $W^{1,3}$. In particular they proved the following properties. 
a) For $H \in C^{1}\left(\mathbb{R}^{3}\right)$, the functional $\mathcal{V}_{H}$ is of class $C^{1}$ on $W^{1,3}$ and the Fréchet differential of $\mathcal{V}_{H}$ at $u \in W^{1,3}$ is given by

$$
d \mathcal{V}_{H}(u) \varphi=\int_{\mathbb{R}^{2}} H(u) \varphi \cdot u_{x} \wedge u_{y} \quad \text { for any } \varphi \in W^{1,3}
$$

and admits a unique continuous and linear extension on $W^{1,3 / 2}$ defined by (2.2). Moreover for every $u \in W^{1,3}$ there exists $\mathcal{V}_{H}^{\prime}(u) \in W^{1,3}$ such that

$$
\left\langle\mathcal{V}_{H}^{\prime}(u), \varphi\right\rangle=\int_{\mathbb{R}^{2}} H(u) \varphi \cdot u_{x} \wedge u_{y} \quad \text { for any } \varphi \in W^{1,3 / 2}
$$

b) For $H \in C^{2}\left(\mathbb{R}^{3}\right)$, the map $\mathcal{V}_{H}^{\prime}: W^{1,3} \rightarrow W^{1,3}$ is of class $C^{1}$ and

$$
\left\langle\mathcal{V}_{H}^{\prime \prime}(u) \cdot \eta, \varphi\right\rangle=\int_{\mathbb{R}^{2}} H(u) \varphi \cdot\left(\eta_{x} \wedge u_{y}+u_{x} \wedge \eta_{y}\right)+\int_{\mathbb{R}^{2}}(\nabla H(u) \cdot \eta) \varphi \cdot\left(u_{x} \wedge u_{y}\right)
$$

$$
\text { for any } u, \eta \in W^{1,3} \text { and } \varphi \in W^{1,3 / 2} \text {. }
$$

Hence for all $u \in W^{1,3}, \mathcal{E}_{\varepsilon}^{\prime}(u) \in W^{1,3}$ and for any $\varphi \in W^{1,3 / 2}$

$$
\left\langle\mathcal{E}_{\varepsilon}^{\prime}(u), \varphi\right\rangle=\int_{\mathbb{R}^{2}} \nabla u \cdot \nabla \varphi+2 \int_{\mathbb{R}^{2}} \varphi \cdot u_{x} \wedge u_{y}+2 \varepsilon \int_{\mathbb{R}^{2}} H(u) \varphi \cdot u_{x} \wedge u_{y}
$$

As remarked in [7], critical points of $\mathcal{E}_{\varepsilon}$ in $W^{1,3}$ give rise to bounded weak solutions to $\left(P_{\varepsilon}\right)$ and hence by the regularity theory for $H$-systems (see [9]) to classical conformal solutions which are $C^{3, \alpha}$ as maps on $\mathbb{S}^{2}$.

The unperturbed problem, i.e. $\left(P_{\varepsilon}\right)$ for $\varepsilon=0$, has a 9-dimensional manifold of solutions given by

$$
Z=\left\{R \omega \circ L_{\lambda, \xi}+p: R \in S O(3), \lambda>0, \xi \in \mathbb{R}^{2}, p \in \mathbb{R}^{3}\right\}
$$

where $L_{\lambda, \xi} z=\lambda(z-\xi)$ (see [11]). In [11] the nondegeneracy condition $T_{u} Z=\operatorname{ker} \mathcal{E}_{0}^{\prime \prime}(u)$ for any $u \in Z$ (where $T_{u} Z$ denotes the tangent space of $Z$ at $u$ ) is proved (see also [8]).

As observed in [7], in performing the finite dimensional reduction, the dependence on the 6-dimensional conformal group can be neglected since any $H$-system is conformally invariant. Hence we look for critical points of $\mathcal{E}_{\varepsilon}$ constrained on a three-dimensional manifold $Z_{\varepsilon}$ just depending on the translation variable $p \in \mathbb{R}^{3}$. 


\section{Proof of Theorem 1.1}

We start by constructing a perturbed manifold which is a natural constraint for $\mathcal{E}_{\varepsilon}$.

Lemma 3.1 Assume $H \in C^{2}\left(\mathbb{R}^{3}\right) \cap L^{\infty}\left(\mathbb{R}^{3}\right)$ and $\nabla H \in L^{\infty}\left(\mathbb{R}^{3}, \mathbb{R}^{3}\right)$. Then there exist $\varepsilon_{0}>0, C_{1}>0$, and a $C^{1}$ map $\eta:\left(-\varepsilon_{0}, \varepsilon_{0}\right) \times \mathbb{R}^{3} \rightarrow W^{1,3}$ such that for any $p \in \mathbb{R}^{3}$ and $\varepsilon \in\left(-\varepsilon_{0}, \varepsilon_{0}\right)$

$$
\begin{aligned}
\mathcal{E}_{\varepsilon}^{\prime}(\omega+p+\eta(\varepsilon, p)) & \in T_{\omega} Z, \\
\eta(\varepsilon, p) & \in\left(T_{\omega} Z\right)^{\perp}, \\
\int_{\mathbb{S}^{2}} \eta(\varepsilon, p) & =0, \\
\|\eta(\varepsilon, p)\|_{W^{1,3}} & \leq C_{1}|\varepsilon| .
\end{aligned}
$$

Moreover if we assume that the limit of $H$ at $\infty$ exists and

$$
\lim _{|p| \rightarrow \infty} H(p)=0
$$

we have that $\eta(\varepsilon, p)$ converges to 0 in $W^{1,3}$ as $|p| \rightarrow \infty$ uniformly with respect to $|\varepsilon|<\varepsilon_{0}$.

Proof. Let us define the map

$$
F=\left(F_{1}, F_{2}\right): \mathbb{R} \times \mathbb{R}^{3} \times W^{1,3} \times \mathbb{R}^{6} \times \mathbb{R}^{3} \rightarrow W^{1,3} \times \mathbb{R}^{6} \times \mathbb{R}^{3}
$$

given by

$$
\left\langle F_{1}(\varepsilon, p, \eta, \lambda, \alpha), \varphi\right\rangle=\left\langle\mathcal{E}_{\varepsilon}^{\prime}(\omega+p+\eta), \varphi\right\rangle-\sum_{i=1}^{6} \lambda_{i} \int_{\mathbb{R}^{2}} \nabla \varphi \cdot \nabla \tau_{i}+\alpha \cdot \int_{\mathbb{S}^{2}} \varphi,
$$

for all $\varphi \in W^{1,3 / 2}$ and

$$
F_{2}(\varepsilon, p, \eta, \lambda, \alpha)=\left(\int_{\mathbb{R}^{2}} \nabla \eta \cdot \nabla \tau_{1}, \ldots, \int_{\mathbb{R}^{2}} \nabla \eta \cdot \nabla \tau_{6}, \int_{\mathbb{S}^{2}} \eta\right)
$$

where $\tau_{1}, \ldots, \tau_{6}$ are chosen in $T_{\omega} Z$ such that

$$
\int_{\mathbb{R}^{2}} \nabla \tau_{i} \cdot \nabla \tau_{j}=\delta_{i j} \quad \text { and } \quad \int_{\mathbb{S}^{2}} \tau_{i}=0 \quad i, j=1, \ldots, 6
$$

so that $T_{\omega} Z$ is spanned by $\tau_{1}, \ldots, \tau_{6}, e_{1}, e_{2}, e_{3}$. It has been proved by Caldiroli and Musina [7] that $F$ is of class $C^{1}$ and that the linear continuous operator

$$
\begin{gathered}
\mathcal{L}: W^{1,3} \times \mathbb{R}^{6} \times \mathbb{R}^{3} \rightarrow W^{1,3} \times \mathbb{R}^{6} \times \mathbb{R}^{3} \\
\mathcal{L}=\frac{\partial F}{\partial(\eta, \lambda, \alpha)}(0, p, 0,0,0)
\end{gathered}
$$


i.e.

$$
\left\langle\mathcal{L}_{1}(v, \mu, \beta), \varphi\right\rangle=\left\langle\mathcal{E}_{0}^{\prime \prime}(\omega) \cdot v, \varphi\right\rangle-\sum_{i=1}^{6} \mu_{i} \int_{\mathbb{R}^{2}} \nabla \varphi \cdot \tau_{i}-\beta \int_{\mathbb{S}^{2}} \varphi
$$

for all $\varphi \in W^{1,3 / 2}$ and

$$
\mathcal{L}_{2}(v, \mu, \beta)=\left(\int_{\mathbb{R}^{2}} \nabla v \cdot \nabla \tau_{1}, \ldots, \int_{\mathbb{R}^{2}} \nabla v \cdot \nabla \tau_{6}, \int_{\mathbb{S}^{2}} v\right)
$$

is invertible. Caldiroli and Musina applied the Implicit Function Theorem to solve the equation $F(\varepsilon, p, \eta, \lambda, \alpha)=0$ locally with respect to the variables $\varepsilon, p$, thus finding a $C^{1}$-function $\eta$ on a neighborhood $\left(-\varepsilon_{0}, \varepsilon_{0}\right) \times B_{R} \subset$ $\mathbb{R} \times \mathbb{R}^{3}$ satisfying (3.1), (3.2), and (3.3). We will use instead the Contraction Mapping Theorem, which allows to prove the existence of such a function $\eta$ globally on $\mathbb{R}^{3}$, thanks to the fact that the operator $\mathcal{L}$ does not depend on $p$ and hence it is invertible uniformly with respect to $p \in \mathbb{R}^{3}$.

We have that $F(\varepsilon, p, \eta, \lambda, \alpha)=0$ if and only if $(\eta, \lambda, \alpha)$ is a fixed point of the map $T_{\varepsilon, p}$ defined as

$$
T_{\varepsilon, p}(\eta, \lambda, \alpha)=-\mathcal{L}^{-1} F(\varepsilon, p, \eta, \lambda, \alpha)+(\eta, \lambda, \alpha) .
$$

To prove the existence of $\eta$ satisfying (3.1), (3.2), and (3.3), it is enough to prove that $T_{\varepsilon, p}$ is a contraction in some ball $B_{\rho}(0)$ with $\rho=\rho(\varepsilon)>0$ independent of $p$, whereas the regularity of $\eta(\varepsilon, p)$ follows from the Implicit Function Theorem.

We have that if $\|\eta\|_{W^{1,3}} \leq \rho$

$$
\begin{aligned}
& \| T_{\varepsilon, p}(\eta, \lambda, \alpha) \|_{W^{1,3} \times \mathbb{R}^{6} \times \mathbb{R}^{3}} \\
& \leq C_{2}\|F(\varepsilon, p, \eta, \lambda, \alpha)-\mathcal{L}(\eta, \lambda, \alpha)\|_{W^{1,3} \times \mathbb{R}^{6} \times \mathbb{R}^{3}} \\
& \leq C_{2}\left\|\mathcal{E}_{\varepsilon}^{\prime}(\omega+p+\eta)-\mathcal{E}_{0}^{\prime \prime}(\omega) \eta\right\|_{W^{1,3}} \\
& \leq C_{2}\left(\left\|\mathcal{E}_{0}^{\prime}(\omega+\eta)-\mathcal{E}_{0}^{\prime \prime}(\omega) \eta\right\|_{W^{1,3}}+2|\varepsilon|\left\|\mathcal{V}_{H}^{\prime}(\omega+p+\eta)\right\|_{W^{1,3}}\right) \\
& \leq C_{2}\left(\int_{0}^{1}\left\|\mathcal{E}_{0}^{\prime \prime}(\omega+t \eta)-\mathcal{E}_{0}^{\prime \prime}(\omega)\right\|_{W^{1,3 / 2}} d t\|\eta\|_{W^{1,3}}\right. \\
&\left.\quad+2|\varepsilon|\left\|\mathcal{V}_{H}^{\prime}(\omega+p+\eta)\right\|_{W^{1,3}}\right) \\
& \quad \sup _{\|\eta\|_{W^{1,3}} \leq \rho}\left\|\mathcal{E}_{0}^{\prime \prime}(\omega+\eta)-\mathcal{E}_{0}^{\prime \prime}(\omega)\right\|_{W^{1,3 / 2}} \\
& \quad+2 C_{2}|\varepsilon| \sup _{\|\eta\|_{W^{1,3}} \leq \rho}\left\|\mathcal{V}_{H}^{\prime}(\omega+p+\eta)\right\|_{W^{1,3}}
\end{aligned}
$$

where $C_{2}=\left\|\mathcal{L}^{-1}\right\|_{\mathcal{L}\left(W^{1,3} \times \mathbb{R}^{6} \times \mathbb{R}^{3}\right)}$. 
For $\left(\eta_{1}, \lambda_{1}, \alpha_{1}\right),\left(\eta_{2}, \lambda_{2}, \alpha_{2}\right) \in B_{\rho}(0) \subset W^{1,3} \times \mathbb{R}^{6} \times \mathbb{R}^{3}$ we have

$$
\begin{aligned}
& \frac{\left\|T_{\varepsilon, p}\left(\eta_{1}, \lambda_{1}, \alpha_{1}\right)-T_{\varepsilon, p}\left(\eta_{2}, \lambda_{2}, \alpha_{2}\right)\right\|_{W^{1,3} \times \mathbb{R}^{6} \times \mathbb{R}^{3}}}{C_{2}\left\|\eta_{1}-\eta_{2}\right\|_{W^{1,3}}} \\
& \leq \frac{\left\|\mathcal{E}_{\varepsilon}^{\prime}\left(\omega+p+\eta_{1}\right)-\mathcal{E}_{\varepsilon}^{\prime}\left(\omega+p+\eta_{2}\right)-\mathcal{E}_{0}^{\prime \prime}(\omega)\left(\eta_{1}-\eta_{2}\right)\right\|_{W^{1,3}}}{C_{2}\left\|\eta_{1}-\eta_{2}\right\|_{W^{1,3}}} \\
& \leq \int_{0}^{1}\left\|\mathcal{E}_{\varepsilon}^{\prime \prime}\left(\omega+p+\eta_{2}+t\left(\eta_{1}-\eta_{2}\right)\right)-\mathcal{E}_{0}^{\prime \prime}(\omega)\right\|_{W^{1,3 / 2}} d t \\
& \leq \int_{0}^{1}\left\|\mathcal{E}_{0}^{\prime \prime}\left(\omega+p+\eta_{2}+t\left(\eta_{1}-\eta_{2}\right)\right)-\mathcal{E}_{0}^{\prime \prime}(\omega)\right\|_{W^{1,3 / 2}} d t \\
& \quad+2|\varepsilon| \int_{0}^{1}\left\|\mathcal{V}_{H}^{\prime \prime}\left(\omega+p+\eta_{2}+t\left(\eta_{1}-\eta_{2}\right)\right)\right\|_{W^{1,3 / 2}} d t \\
& \leq \sup _{\|\eta\|_{W^{1,3}} \leq 3 \rho}\left\|\mathcal{E}_{0}^{\prime \prime}(\omega+\eta)-\mathcal{E}_{0}^{\prime \prime}(\omega)\right\|_{W^{1,3 / 2}}+2|\varepsilon|_{\|\eta\|_{W^{1,3} \leq 3 \rho}}\left\|\mathcal{V}_{H}^{\prime \prime}(\omega+p+\eta)\right\|_{W^{1,3 / 2}} .
\end{aligned}
$$

From (2.3), (2.4), and the Hölder inequality it follows that there exists a positive constant $C_{3}$ such that for any $\eta \in W^{1,3}, p \in \mathbb{R}^{3}$

$$
\left\|\mathcal{V}_{H}^{\prime}(\omega+p+\eta)\right\|_{W^{1,3}} \leq C_{3}\left[\left(\int_{\mathbb{R}^{2}}|H(\omega+p+\eta)|^{3 / 2}|\nabla \omega|^{3} \mu^{-1}\right)^{2 / 3}+\|\eta\|_{W^{1,3}}^{2}\right]
$$

and

$$
\begin{aligned}
\left\|\mathcal{V}_{H}^{\prime \prime}(\omega+p+\eta)\right\|_{W^{1,3 / 2}} \leq C_{3} & {\left[\left(\int_{\mathbb{R}^{2}}|H(\omega+p+\eta)|^{2}|\nabla(\omega+\eta)|^{2}\right)^{1 / 2}\right.} \\
& \left.+\left(\int_{\mathbb{R}^{2}}|\nabla H(\omega+p+\eta)|^{3 / 2}|\nabla(\omega+\eta)|^{3} \mu^{-1}\right)^{2 / 3}\right] .
\end{aligned}
$$

Choosing $\rho_{0}>0$ such that

$$
C_{2} \sup _{\|\eta\|_{W^{1,3}} \leq 3 \rho_{0}}\left\|\mathcal{E}_{0}^{\prime \prime}(\omega+\eta)-\mathcal{E}_{0}^{\prime \prime}(\omega)\right\|_{W^{1,3 / 2}}<\frac{1}{2}
$$

and $\varepsilon_{0}>0$ such that

$$
\begin{aligned}
& 8 C_{2} C_{3} \varepsilon_{0}\|H\|_{L^{\infty}\left(\mathbb{R}^{3}\right)}\|\omega\|_{W^{1,3}}^{2}<\min \left\{1, \rho_{0}, \frac{1}{8 C_{2} C_{3} \varepsilon_{0}}\right\},
\end{aligned}
$$

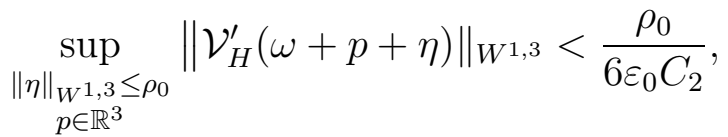

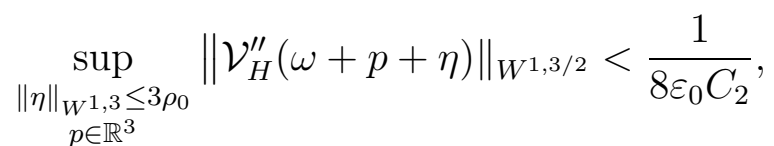

we obtain that $T_{\varepsilon, p}$ maps the ball $\overline{B_{\rho_{0}}(0)}$ into itself for any $|\varepsilon|<\varepsilon_{0}, p \in \mathbb{R}^{3}$, and is a contraction there. 
Hence it has a unique fixed point $(\eta(\varepsilon, p), \lambda(\varepsilon, p), \alpha(\varepsilon, p)) \in \overline{B_{\rho_{0}}(0)}$. From (3.6) we have that the following property holds

(*) $T_{\varepsilon, p}$ maps a ball $\overline{B_{\rho}(0)} \subset W^{1,3} \times \mathbb{R}^{6} \times \mathbb{R}^{3}$ into itself whenever $\rho \leq \rho_{0}$ and $\rho>4|\varepsilon| C_{2} \sup _{\|\eta\|_{W^{1,3} \leq \rho}}\left\|\mathcal{V}_{H}^{\prime}(\omega+p+\eta)\right\|_{W^{1,3}}$.

In particular let us set

$$
\rho_{\varepsilon}=5|\varepsilon| C_{2} \sup _{\substack{\|\eta\|_{W^{1,3} \leq \rho_{0}} \\ p \in \mathbb{R}^{3}}}\left\|\mathcal{V}_{H}^{\prime}(\omega+p+\eta)\right\|_{W^{1,3}}
$$

In view of (3.10) and (3.12), we have that for any $|\varepsilon|<\varepsilon_{0}$ and for any $p \in \mathbb{R}^{3}$

$$
\rho_{\varepsilon} \leq \rho_{0} \quad \text { and } \quad \rho_{\varepsilon}>4|\varepsilon| C_{2} \sup _{\|\eta\|_{W^{1,3} \leq \rho_{\varepsilon}}}\left\|\mathcal{V}_{H}^{\prime}(\omega+p+\eta)\right\|_{W^{1,3}}
$$

so that, due to $(*), T_{\varepsilon, p}$ maps $\overline{B_{\rho_{\varepsilon}}(0)}$ into itself. From the uniqueness of the fixed point we have that for any $|\varepsilon|<\varepsilon_{0}$ and $p \in \mathbb{R}^{3}$

$$
\|(\eta(\varepsilon, p), \lambda(\varepsilon, p), \alpha(\varepsilon, p))\|_{W^{1,3} \times \mathbb{R}^{6} \times \mathbb{R}^{3}} \leq \rho_{\varepsilon} \leq C_{1}|\varepsilon|
$$

for some positive constant $C_{1}$ independent of $p$ and hence $\|\eta(\varepsilon, p)\|_{W^{1,3}} \leq$ $\rho_{\varepsilon} \leq C_{1}|\varepsilon|$ thus proving (3.4). Assume now (3.5) and set for any $p \in \mathbb{R}^{3}$

$$
\rho_{p}=8 C_{2} C_{3} \varepsilon_{0}\left(\int_{\mathbb{R}^{2}|q-p| \leq 1+C_{0}} \sup _{\left.|H(q)|^{3 / 2}|\nabla \omega|^{3} \mu^{-1}\right)^{2 / 3}}\right.
$$

where $C_{0}$ is a positive constant such that $\|u\|_{L^{\infty}} \leq C_{0}\|u\|_{W^{1,3}}$ for any $u \in W^{1,3}$. From (3.9) we have that

$$
\rho_{p}<\min \left\{1, \rho_{0}, \frac{1}{8 C_{2} C_{3} \varepsilon_{0}}\right\} .
$$

Hence, due to (3.7), we have that for $|\varepsilon|<\varepsilon_{0}$ and $\|\eta\|_{W^{1,3}} \leq \rho_{p}$

$$
\begin{aligned}
& 4|\varepsilon| C_{2}\left\|\mathcal{V}_{H}^{\prime}(\omega+p+\eta)\right\|_{W^{1,3}} \\
& \quad \leq 4 \varepsilon_{0} C_{2} C_{3}\left(\int_{\mathbb{R}^{2}|q-p| \leq 1+C_{0}} \sup _{\left.|H(q)|^{3 / 2}|\nabla \omega|^{3} \mu^{-1}\right)^{2 / 3}+4 \varepsilon_{0} C_{2} C_{3} \rho_{p}^{2}<\rho_{p} .}\right.
\end{aligned}
$$

From $(*)$ and the uniqueness of the fixed point, we deduce that

$$
\|\eta(\varepsilon, p)\|_{W^{1,3}} \leq \rho_{p}
$$

for any $|\varepsilon|<\varepsilon_{0}$ and $p \in \mathbb{R}^{3}$. On the other hand, since $H$ vanishes at $\infty$, by the definition of $\rho_{p}$ we have that $\rho_{p} \rightarrow 0$ as $|p| \rightarrow \infty$, hence

$$
\lim _{|p| \rightarrow \infty} \eta(\varepsilon, p)=0 \quad \text { in } W^{1,3} \text { uniformly for }|\varepsilon|<\varepsilon_{0} .
$$

The proof of Lemma 3.1 is now complete. 
Remark 3.2 The map $\eta$ given in Lemma 3.1 satisfies $\left\langle\mathcal{E}_{\varepsilon}^{\prime}(\omega+p+\eta(\varepsilon, p)), \varphi\right\rangle-\sum_{i=1}^{6} \lambda_{i}(\varepsilon, p) \int_{\mathbb{R}^{2}} \nabla \varphi \cdot \nabla \tau_{i}+\alpha(\varepsilon, p) \cdot \int_{\mathbb{S}^{2}} \varphi, \quad \forall \varphi \in W^{1,3 / 2}$ where $(\eta(\varepsilon, p), \lambda(\varepsilon, p), \alpha(\varepsilon, p)) \in \overline{B_{\rho_{\varepsilon}}(0)} \subset W^{1,3} \times \mathbb{R}^{6} \times \mathbb{R}^{3}$ being $\rho_{\varepsilon}$ given in (3.12), hence

$$
\begin{aligned}
\int_{\mathbb{R}^{2}} \nabla(\omega & +\eta(\varepsilon, p)) \cdot \nabla \varphi+2 \int_{\mathbb{R}^{2}} \varphi \cdot(\omega+\eta(\varepsilon, p))_{x} \wedge(\omega+\eta(\varepsilon, p))_{y} \\
+2 \varepsilon & \int_{\mathbb{R}^{2}} H(\omega+p+\eta(\varepsilon, p)) \varphi \cdot(\omega+\eta(\varepsilon, p))_{x} \wedge(\omega+\eta(\varepsilon, p))_{y} \\
& =\sum_{i=1}^{6} \lambda_{i}(\varepsilon, p) \int_{\mathbb{R}^{2}} \nabla \varphi \cdot \nabla \tau_{i}-\alpha(\varepsilon, p) \cdot \int_{\mathbb{S}^{2}} \varphi, \quad \forall \varphi \in W^{1,3 / 2},
\end{aligned}
$$

i.e. $\eta(\varepsilon, p)$ satisfies the equation

$$
\Delta \eta(\varepsilon, p)=F(\varepsilon, p)
$$

where

$$
\begin{aligned}
F(\varepsilon, p)= & 2(\omega+\eta(\varepsilon, p))_{x} \wedge(\omega+\eta(\varepsilon, p))_{y}-2 \omega_{x} \wedge \omega_{y}+\lambda(\varepsilon, p) \cdot \Delta \tau \\
& -\alpha(\varepsilon, p) \mu^{2}+2 \varepsilon H(\omega+p+\eta(\varepsilon, p))(\omega+\eta(\varepsilon, p))_{x} \wedge(\omega+\eta(\varepsilon, p))_{y}
\end{aligned}
$$

in $\mathbb{R}^{2}$. Since $F(\varepsilon, p) \in L^{3 / 2}$ and, in view of (3.4) and (3.13), $F(\varepsilon, p) \rightarrow 0$ in $L^{3 / 2}$ as $\varepsilon \rightarrow 0$ uniformly with respect to $p$, by regularity we have that

$$
\eta(\varepsilon, p) \in W^{2,3 / 2} \quad \text { and } \quad \eta(\varepsilon, p) \rightarrow 0 \text { in } W^{2,3 / 2}
$$

hence, by Sobolev embeddings, $F(\varepsilon, p) \in L^{3}$ and $F(\varepsilon, p) \rightarrow 0$ in $L^{3}$ as $\varepsilon \rightarrow 0$ uniformly with respect to $p$. Again by regularity

$$
\eta(\varepsilon, p) \in W^{2,3} \quad \text { and } \quad \eta(\varepsilon, p) \rightarrow 0 \text { in } W^{2,3}
$$

hence $\eta(\varepsilon, p) \in C^{1,1 / 3}$ and

$$
\eta(\varepsilon, p) \rightarrow 0 \quad \text { in } C^{1,1 / 3} \text { as } \varepsilon \rightarrow 0 \text { uniformly with respect to } p \text {. }
$$

For any $\varepsilon \in\left(-\varepsilon_{0}, \varepsilon_{0}\right)$, let us define the perturbed manifold

$$
Z_{\varepsilon}:=\left\{\omega+p+\eta(\varepsilon, p): p \in \mathbb{R}^{3}\right\} .
$$

From [7], we have that $Z_{\varepsilon}$ is a natural constraint for $\mathcal{E}_{\varepsilon}$, namely any critical point $p \in \mathbb{R}^{3}$ of the functional

$$
\Phi_{\varepsilon}: \mathbb{R}^{3} \rightarrow \mathbb{R}, \quad \Phi_{\varepsilon}(p)=\mathcal{E}_{\varepsilon}(\omega+p+\eta(\varepsilon, p))
$$

gives rise to a critical point $u_{\varepsilon}=\omega+p+\eta(\varepsilon, p)$ of $\mathcal{E}_{\varepsilon}$. 
Proposition 3.3 Assume $H \in C^{2}\left(\mathbb{R}^{3}\right), \nabla H \in L^{\infty}\left(\mathbb{R}^{3}, \mathbb{R}^{3}\right)$, and

$$
\lim _{|p| \rightarrow \infty} H(p)=0
$$

Then for any $|\varepsilon|<\varepsilon_{0}$

$$
\lim _{|p| \rightarrow \infty} \Phi_{\varepsilon}(p)=\text { const }=\mathcal{E}_{0}(\omega) .
$$

Proof. We have that

$$
\begin{aligned}
\Phi_{\varepsilon}(p)= & \mathcal{E}_{\varepsilon}(\omega+p+\eta(\varepsilon, p)) \\
= & \mathcal{E}_{0}(\omega+p+\eta(\varepsilon, p))+2 \varepsilon \mathcal{V}_{H}(\omega+p+\eta(\varepsilon, p)) \\
=\frac{1}{2} & \int_{\mathbb{R}^{2}}|\nabla \omega|^{2}+\frac{1}{2} \int_{\mathbb{R}^{2}}|\nabla \eta(\varepsilon, p)|^{2}+\int_{\mathbb{R}^{2}} \nabla \omega \cdot \nabla \eta(\varepsilon, p) \\
& +\frac{2}{3} \int_{\mathbb{R}^{2}}(\omega+p+\eta(\varepsilon, p)) \cdot(\omega+\eta(\varepsilon, p))_{x} \wedge(\omega+\eta(\varepsilon, p))_{y} \\
& +2 \varepsilon\left[\mathcal{V}_{H}(\omega+p)+\left\langle\mathcal{V}_{H}^{\prime}(\omega+p), \eta(\varepsilon, p)\right\rangle+o\left(\|\eta(\varepsilon, p)\|_{W^{1,3}}\right)\right] \\
=\frac{1}{2} & \int_{\mathbb{R}^{2}}|\nabla \omega|^{2}+\frac{2}{3} \int_{\mathbb{R}^{2}} \omega \cdot \omega_{x} \wedge \omega_{y}+\frac{1}{2} \int_{\mathbb{R}^{2}}|\nabla \eta(\varepsilon, p)|^{2} \\
& +\int_{\mathbb{R}^{2}} \nabla \omega \cdot \nabla \eta(\varepsilon, p)+\frac{2}{3} \int_{\mathbb{R}^{2}} \omega \cdot\left(\omega_{x} \wedge \eta(\varepsilon, p)_{y}+\eta(\varepsilon, p)_{x} \wedge \omega_{y}\right) \\
& +\frac{2}{3} \int_{\mathbb{R}^{2}} \omega \cdot \eta(\varepsilon, p)_{x} \wedge \eta(\varepsilon, p)_{y} \\
& +\frac{2}{3} \int_{\mathbb{R}^{2}} \eta(\varepsilon, p) \cdot(\omega+\eta(\varepsilon, p))_{x} \wedge(\omega+\eta(\varepsilon, p))_{y} \\
& +2 \varepsilon \mathcal{V}_{H}(\omega+p)+2 \varepsilon\left\langle\mathcal{V}_{H}^{\prime}(\omega+p), \eta(\varepsilon, p)\right\rangle+2 \varepsilon o\left(\|\eta(\varepsilon, p)\|_{W^{1,3}}\right)
\end{aligned}
$$

where we have used the fact that

$$
\int_{\mathbb{R}^{2}} p \cdot u_{x} \wedge u_{y}=0 \quad \forall p \in \mathbb{R}^{3}, u \in W^{1,3},
$$

(see [7], Lemma A.3). Notice that from Lemma 3.1 we have that

$$
\begin{aligned}
\int_{\mathbb{R}^{2}}|\nabla \eta(\varepsilon, p)|^{2} & \leq \sqrt[3]{4 \pi}\|\eta(\varepsilon, p)\|_{W^{1,3}}^{2} \underset{|p| \rightarrow \infty}{\longrightarrow} 0 \\
\left|\int_{\mathbb{R}^{2}} \nabla \omega \cdot \nabla \eta(\varepsilon, p)\right| & \leq \sqrt[6]{4 \pi}\left(\int_{\mathbb{R}^{2}}|\nabla \omega|^{2}\right)^{1 / 2}\|\eta(\varepsilon, p)\|_{W^{1,3}} \underset{|p| \rightarrow \infty}{\longrightarrow} 0
\end{aligned}
$$


and, by the Hölder inequality and Lemma 3.1,

$$
\begin{gathered}
\left|\int_{\mathbb{R}^{2}} \omega \cdot\left(\omega_{x} \wedge \eta(\varepsilon, p)_{y}+\eta(\varepsilon, p)_{x} \wedge \omega_{y}\right)\right| \leq 2\|\omega\|_{W^{1,3}}^{2}\|\eta(\varepsilon, p)\|_{W^{1,3}} \underset{|p| \rightarrow \infty}{\longrightarrow} 0 \\
\left|\int_{\mathbb{R}^{2}} \omega \cdot\left(\eta(\varepsilon, p)_{x} \wedge \eta(\varepsilon, p)_{y}\right)\right| \leq\|\omega\|_{W^{1,3}}\|\eta(\varepsilon, p)\|_{W^{1,3}}^{2} \underset{|p| \rightarrow \infty}{\longrightarrow} 0,
\end{gathered}
$$

and

$$
\begin{aligned}
& \mid \int_{\mathbb{R}^{2}} \eta(\varepsilon, p) \cdot(\omega+\eta(\varepsilon, p))_{x} \wedge(\omega+\eta(\varepsilon, p))_{y} \mid \\
& \leq\|\omega+\eta(\varepsilon, p)\|_{W^{1,3}}^{2}\|\eta(\varepsilon, p)\|_{W^{1,3}} \underset{|p| \rightarrow \infty}{\longrightarrow} 0 .
\end{aligned}
$$

Moreover the Gauss-Green Theorem yields

$$
\mathcal{V}_{H}(\omega+p)=-\int_{B_{1}} H(\xi+p) d \xi
$$

so that by the Dominated Convergence Theorem we have that

$$
\lim _{|p| \rightarrow \infty} \mathcal{V}_{H}(\omega+p)=0 .
$$

From (2.3), Hölder's inequality, and Lemma 3.1, we have that

$$
\begin{aligned}
\left|\left\langle\mathcal{V}_{H}^{\prime}(\omega+p), \eta(\varepsilon, p)\right\rangle\right| & =\left|\int_{\mathbb{R}^{2}} H(\omega+p) \eta(\varepsilon, p) \cdot \omega_{x} \wedge \omega_{y}\right| \\
& \leq\|H\|_{L^{\infty}\left(\mathbb{R}^{3}\right)}\|\omega\|_{W^{1,3}}^{2}\|\eta(\varepsilon, p)\|_{W^{1,3}} \underset{|p| \rightarrow \infty}{\longrightarrow} 0 .
\end{aligned}
$$

From (3.15)-(3.22), it follows that

$$
\lim _{|p| \rightarrow \infty} \Phi_{\varepsilon}(p)=\frac{1}{2} \int_{\mathbb{R}^{2}}|\nabla \omega|^{2}+\frac{2}{3} \int_{\mathbb{R}^{2}} \omega \cdot \omega_{x} \wedge \omega_{y}=\mathcal{E}_{0}(\omega) .
$$

The proposition is thereby proved.

Proof of Theorem 1.1. As already observed at the beginning of Section 2, it is not restrictive to take $H_{0}=1$. From Proposition 3.3 it follows that for $|\varepsilon|<\varepsilon_{0}$ either $\Phi_{\varepsilon}$ is constant (and hence we have infinitely many critical points) or it has a global maximum or minimum point. In any case $\Phi_{\varepsilon}$ has a critical point. Since $Z_{\varepsilon}$ is a natural constraint for $\mathcal{E}_{\varepsilon}$, we deduce the existence of a critical point of $\mathcal{E}_{\varepsilon}$ for $|\varepsilon|<\varepsilon_{0}$ and hence of a solution to $\left(P_{\varepsilon}\right)$. The $H_{\varepsilon}$-bubble $\omega_{\varepsilon}$ found in this way is of the form $\omega+p^{\varepsilon}+\eta\left(\varepsilon, p^{\varepsilon}\right)$ for some $p^{\varepsilon} \in \mathbb{R}^{3}$ where $\eta$ is as in Lemma 3.1. Remark 3.2 yields that $\omega_{\varepsilon}$ is closed in $C^{1,1 / 3}\left(\mathbb{S}^{2}, \mathbb{R}^{3}\right)$-norm to the manifold $\left\{\omega+p: p \in \mathbb{R}^{3}\right\}$ for $\varepsilon$ small. Since $\omega$ has no branch points, we deduce that $\omega_{\varepsilon}$ has no branch points. 
To prove Theorems 1.2 and 1.4, we need the following expansion for $\Phi_{\varepsilon}$ :

$$
\Phi_{\varepsilon}(p)=\mathcal{E}_{0}(\omega)-2 \varepsilon \Gamma(p)+O\left(\varepsilon^{2}\right)
$$

as $\varepsilon \rightarrow 0$ uniformly in $p \in \mathbb{R}^{3}$ (see [7]).

Proof of Theorem 1.2. Let $\varepsilon>0$ small. Assumption (H4) implies that $\Gamma(0)>0$ and hence from (3.23) we have that for $\varepsilon$ small

$$
\Phi_{\varepsilon}(0)<\mathcal{E}_{0}(\omega)
$$

whereas from assumption $(H 3)$ we have that $\operatorname{Hess} \Gamma(0)$ is positive definite so that $\Gamma$ has a strict local minimum in 0 and hence from $(3.23) \Phi_{\varepsilon}$ has a strict local maximum in $B_{r}(0)$ for some $r>0$ such that

$$
\Phi_{\varepsilon}(p)<\Phi_{\varepsilon}(0)-c_{\varepsilon}<\mathcal{E}_{0}(\omega)
$$

for $|p|=r$, where $c_{\varepsilon}$ is some positive constant depending on $\varepsilon$. In particu$\operatorname{lar} \Phi_{\varepsilon}$ has a mountain pass geometry. Moreover by Theorem 1.1,

$$
\Phi_{\varepsilon}(p) \rightarrow \mathcal{E}_{0}(\omega) \text { as } \quad|p| \rightarrow \infty
$$

and so $\Phi_{\varepsilon}$ must have a global minimum point. If the minimum point and the mountain pass point coincide then $\Phi_{\varepsilon}$ has infinitely many critical points. Otherwise $\Phi_{\varepsilon}$ has at least three critical points: a local maximum point, a global minimum point, and a mountain pass. If $\varepsilon<0$ we find the inverse inequalities and hence we find that $\Phi_{\varepsilon}$ has a local minimum point, a global maximum point, and a mountain pass. As a consequence $\left(P_{\varepsilon}\right)$ has at least three solutions provided $|\varepsilon|$ is sufficiently small.

As observed in Remark 1.3, if $H_{1}(0)>0$ and Hess $H_{1}(0)$ is positive definite, by continuity we have that for $H_{0}$ sufficiently large $\Gamma(0)>0$ and Hess $\Gamma(0)$ is positive definite, so that we can still prove the existence of three solutions arguing as above.

Proof of Theorem 1.4. Assumption $(H 5)$ implies that $\Gamma\left(p_{1}\right)>0$ and $\Gamma\left(p_{2}\right)<0$. Since

$$
\Phi_{\varepsilon}(p)=\mathcal{E}_{0}(\omega)+2 \varepsilon(-\Gamma(p)+o(1)) \quad \text { as } \varepsilon \rightarrow 0,
$$

we have for $\varepsilon$ sufficiently small

$$
\Phi_{\varepsilon}\left(p_{1}\right)<\mathcal{E}_{0}(\omega) \text { and } \Phi_{\varepsilon}\left(p_{2}\right)>\mathcal{E}_{0}(\omega)
$$

if $\varepsilon>0$ and the inverse inequalities if $\varepsilon<0$. Since by Theorem 1.1

$$
\Phi_{\varepsilon}(p) \rightarrow \mathcal{E}_{0}(\omega) \quad \text { as } \quad|p| \rightarrow \infty
$$


we conclude that $\Phi_{\varepsilon}$ must have a global maximum point and a global minimum point in $\mathbb{R}^{3}$. Since $Z_{\varepsilon}$ is a natural constraint for $\mathcal{E}_{\varepsilon}$, we deduce the existence of two critical points of $\mathcal{E}_{\varepsilon}$ for $|\varepsilon|$ sufficiently small and hence of two solutions to $\left(P_{\varepsilon}\right)$.

As observed in Remark 1.5, if $H_{1}\left(p_{1}\right)>0$ and $H_{1}\left(p_{2}\right)<0$, by continuity we have that for $H_{0}$ sufficiently large $\Gamma\left(p_{1}\right)>0$ and $\Gamma\left(p_{2}\right)<0$, so that we can still prove the existence of two solutions arguing as above.

\section{References}

[1] Ambrosetti, A. And Badiale, M.: Homoclinics: Poincaré-Melnikov type results via a variational approach. Ann. Inst. H. Poincaré Anal. Non Linéaire 15 (1998), no. 2, 233-252.

[2] Ambrosetti, A. And Badiale. M.: Variational perturbative methods and bifurcation of bound states from the essential spectrum. Proc. Roy. Soc. Edinburgh Sect. A 128 (1998), 1131-1161.

[3] Brezis, H. And Coron, J. M.: Multiple solutions of $H$-systems and Rellich's conjecture. Comm. Pure Appl. Math. 37 (1984), no. 2, 149-187.

[4] Brezis, H. and Coron, J. M.: Convergence of solutions of $H$-systems or how to blow bubbles. Arch. Rational Mech. Anal. 89 (1985), no. 1, 21-56.

[5] Caldiroli, P. and Musina, R.: Existence of minimal $H$-bubbles. Commun. Contemp. Math. 4 (2002), no. 2, 177-210.

[6] Caldiroli, P. and Musina, R.: Existence of $H$-bubbles in a perturbative setting. Rev. Mat. Iberoamericana 20 (2004), no. 2, 611-626.

[7] Caldiroli, P. And Musina, R.: $H$-bubbles in a perturbative setting: the finite-dimensional reduction method. Duke Math. J. 122 (2004), no. 3, $457-484$.

[8] Chanillo, S. and Malchiodi, A.: Asymptotic Morse theory for the equation $\Delta u=2 u_{x} \wedge u_{y}$. Comm. Anal. Geom. 13 (2005), no. 1, 187-251.

[9] Grüter, M.: Regularity of weak $H$-surfaces. J. Reine Angew. Math. 329 (1981), 1-15.

[10] Hildebrandt, S.: Randwertprobleme für Flächen mit vorgeschriebener mittlerer Krümmung und Anwendungen auf die Kapillaritätstheorie. I: Fest vorgegebener Rand. Math. Z. 112 (1969), 205-213.

[11] Isobe, T.: On the asymptotic analysis of $H$-systems, I: asymptotic behavior of large solutions. Adv. Differential Equations 6 (2001), no. 5, 513-546.

[12] Jаковошsкy, N.: A perturbation result concerning a second solution to the Dirichlet problem for the equation of the prescribed mean curvature. $J$. Reine Angew. Math. 457 (1994), 1-21.

[13] Jakовоwsky, N.: Multiple surfaces of non-constant mean curvature. Math. Z. 217 (1994), no. 3, 497-512. 
[14] Steffen, K.: On the existence of surfaces with prescribed mean curvature and boundary. Math. Z. 146 (1976), no. 2, 113-135.

[15] Struwe, M.: Large H-surface via the mountain-pass lemma. Math. Ann. 270 (1985), no. 3, 441-459.

[16] Struwe, M.: Plateau's problem and the calculus of variations. Mathematical Notes 35. Princeton University Press, Princeton, NJ, 1988.

Recibido: 29 de enero de 2003.

Veronica Felli

Dipartimento di Matematica e Applicazioni Università di Milano-Bicocca

Via Cozzi 53

20125 Milano, Italy

veronica.felli@unimib.it

The author wishes to thank Professor A. Ambrosetti and Professor R. Musina for many helpful suggestions. Supported by MIUR, national project "Variational Methods and Nonlinear Differential Equations". 